\title{
Correction to: Spatiotemporal Modeling of Cancer Immunotherapy
}

\section{Correction to:}

\section{W. E. Schiesser, Spatiotemporal Modeling}

of Cancer Immunotherapy, https://doi.org/10.1007/978-3-030-19080-4

The author had missed to provide $\mathrm{R}$ routines associated with chapters $2-5$. Now the files are uploaded to springer sites and appropriate links have been inserted in the respective chapters.

\footnotetext{
The updated online version of these chapters can be found at https://doi.org/10.1007/978-3-030-19080-4_2 https://doi.org/10.1007/978-3-030-19080-4_3 https://doi.org/10.1007/978-3-030-19080-4_4 https://doi.org/10.1007/978-3-030-19080-4_5
} 